๑ Open Access Full Text Article

\title{
Assessment of ischemia in acute central retinal vein occlusion from inner retinal reflectivity on spectral domain optical coherence tomography
}

This article was published in the following Dove Press journal:

Clinical Ophthalmology

22 December 2016

Number of times this article has been viewed

\section{David J Browning \\ Omar S Punjabi \\ Chong Lee}

Department of Ophthalmology, Charlotte Eye, Ear, Nose and Throat Associates, P.A., Charlotte, NC, USA
Correspondence: David J Browning Department of Ophthalmology, Charlotte Eye, Ear, Nose and Throat Associates, P.A., 6035 Fairview Rd, Charlotte, NC 28210, USA

Tel +l 7042953180

Fax +l 7042953186

Email dbrowning@ceenta.com
Purpose: To determine the relationship between different spectral domain optical coherence tomography (SD-OCT) signs of retinal ischemia in acute central retinal vein occlusion (CRVO) and whether they predict anterior segment neovascularization (ASNV).

Design: Retrospective, observational study.

Subjects: Thirty-nine consecutive patients with acute CRVO and 12 months of follow-up.

Methods: We graded baseline SD-OCTs for increased reflectivity of the inner retina, loss of definition of inner retinal layers, presence of a prominent middle-limiting membrane (p-MLM) sign, and presence of paracentral acute middle maculopathy (PAMM). Graders were masked with respect to all clinical information.

Results: The intraclass correlation coefficients (ICCs) of grading-regrading by graders 1 and 2 were $0.8104,95 \%$ confidence interval (CI) $(0.6686,0.8956)$, and $0.7986,95 \%$ CI $(0.6475$, 0.8892 ), respectively. The intragrader coefficients of repeatability (COR) for graders 1 and 2 were 0.94 and 0.92 , respectively. The ICC of graders 1 compared with 2 was $0.8039,95 \%$ CI $(0.6544,0.8916)$. The intergrader COR was 0.80 . SD-OCT grades of baseline ischemia were not associated with baseline visual acuity (VA), central subfield mean thickness (CSMT), or relative afferent pupillary defect; 12-month VA, CSMT, change in VA, change in CSMT, number of antivascular endothelial growth factor injections or corticosteroid injections, or proportion of eyes developing ASNV. SD-OCT grades of ischemia did not correlate with the proportion of eyes having the p-MLM sign or PAMM. PAMM and p-MLM are milder signs of ischemia than increased reflectivity of the inner retinal layers. Eyes with PAMM can evolve, losing PAMM and gaining the p-MLM sign.

Conclusion: Grading of ischemia from SD-OCT in acute CRVO was repeatable within graders and reproducible across graders for the graders in this study. SD-OCT signs of ischemia are not correlated with each other and do not reliably predict subsequent ASNV. Close monitoring of eyes with acute CRVO continues to be the safest method to avoid missing ASNV and neovascular glaucoma.

Keywords: central retinal vein occlusion, spectral domain optical coherence tomography, ischemia, prominent middle-limiting membrane sign, paracentral acute middle maculopathy, rubeosis iris, anterior segment neovascularization, iris neovascularization

\section{Introduction}

Assessing ischemia in acute central retinal vein occlusion (CRVO) has attracted clinical efforts for 50 years in an attempt to predict the development of anterior segment neovascularization (ASNV) and neovascular glaucoma (NVG) and to guide the frequency of follow-up. Approaches have included stratification by bestcorrected visual acuity (BCVA), measurement of the area of capillary nonperfusion 
on fluorescein angiography (FA), quantitation of the relative afferent pupillary defect (RAPD), assessment of the scotoma size on Goldmann perimetry, and measurement of various indices on global electroretinography (ERG). ${ }^{1-3}$ Recently, spectral domain optical coherence tomography (SD-OCT) was found to be useful in this regard. The "prominent middlelimiting membrane sign" (p-MLM sign) was described as a hyperreflective line at the inner synaptic portion of the outer plexiform layer occurring in $28 \%$ of cases and associated with ischemic CRVO diagnosed from overall clinical impression based on BCVA, fundus examination, RAPD, and FA made independent of SD-OCT grading. ${ }^{4}$ In addition, paramacular acute middle maculopathy, an SD-OCT sign of ischemia in the deeper capillary bed of the inner retina, has been observed in CRVO among other conditions. ${ }^{5}$

We have described other SD-OCT associations with ischemia in acute CRVO, including the loss of definition of the boundaries between the inner retinal lamina and increased reflectivity of the inner retina. ${ }^{6}$ In this study, we addressed the question whether these signs have value in predicting visual outcome and development of ASNV in eyes with acute CRVO.

\section{Methods}

This was a retrospective study of 39 consecutive patients with acute CRVO irrespective of the presence or absence of macular edema who had baseline and follow-up SDOCTs and at least 12-month follow-up. Acute CRVO was defined as an episode in which the patient noted a change in vision leading to a visit to the ophthalmologist together with a fundus appearance compatible with CRVO. No patient received treatment before capture of the SD-OCT images. Baseline data extracted from the clinical charts included demographic information, presence of diabetes mellitus, hypertension, and preexisting primary open angle glaucoma. Follow-up information included development of ASNV; best measured visual acuity (VA) using the patient's correction and pinhole; and presence or absence of a RAPD; interventions including antivascular endothelial growth factor injections (anti-VEGF), intravitreal corticosteroid injections, laser panretinal photocoagulation, and incisional glaucoma procedures; and measurements of SD-OCT central subfield mean thickness (CSMT).

The level of ischemia on SD-OCT images was graded as none to mild (grade 1), moderate (grade 2), and severe (grade 3). The standard images used in grading the SD-OCTs are shown in Figures 1-3. Grading of ischemia from the SD-OCT was based on whether the inner retinal layers could be delineated and on the reflectivity of these layers. The presence and quantity of macular edema, intraretinal cysts, or subretinal fluid had no bearing on the grade of ischemia. When there was concomitant intraretinal hemorrhage, the grading was done based on a zone without intraretinal hemorrhage if one was present, but if intraretinal hemorrhage was confluent, the inner retina was hyperreflective and delineation of layers was obscured by the hemorrhage, such cases were graded as severe ischemia.

Intragrader repeatability and intergrader agreement were assessed by intraclass correlation coefficients (ICCs) and Bland-Altman analysis with the calculation of coefficients of repeatability (COR). ${ }^{7}$ Statistical analyses were performed using JMP software (version 4.0, SAS Corporation, Cary, NC, USA) and MedCalc statistical software version 15.11.1 (MedCalc Software bvba, Ostend, Belgium).

Ischemia grades for baseline SD-OCTs were assigned independently by two experienced retina specialists twice with several weeks between gradings to allow intra- and intergrader agreements to be determined. Correlations of baseline ischemia grades with proportions of baseline p-MLM and paracentral acute middle maculopathy (PAMM) signs were calculated.

Waiver of written informed consent from participants and waiver of patients' data privacy and security provisions according to Health Insurance Portability and Accountability Act of 1996 (HIPAA) were approved by the Presbyterian Hospital Institutional Review Board (ID 16-455).

\section{Results}

The ICC of grading-regrading by graders 1 and 2 was $0.8104,95 \%$ confidence interval (CI) $(0.6686,0.8956)$, and $0.7986,95 \%$ CI $(0.6475,0.8892)$, respectively. The intragrader COR for graders 1 and 2 was 0.94 and 0.92 , respectively. The ICC of grader 1 compared with 2 was $0.8039,95 \%$ CI $(0.6544,0.8916)$. The intergrader COR was 0.80 . Bland-Altman plots for intragrader repeatability and intergrader reproducibility are shown in Figure 4. There was no evidence of fixed or proportional bias between graders.

Of the 39 eyes, the distribution was 13, 17, and 9 for mild, moderate, and severe grades of ischemia at baseline, respectively. Demographic information on the subjects is shown in Table 1. There were no statistically significant differences in age, gender, or prevalence of hypertension, diabetes, or primary open angle glaucoma across the grades of ischemia as graded from baseline SD-OCTs. The median duration of symptoms before examination was 2 weeks, interquartile range 1-6 weeks. 

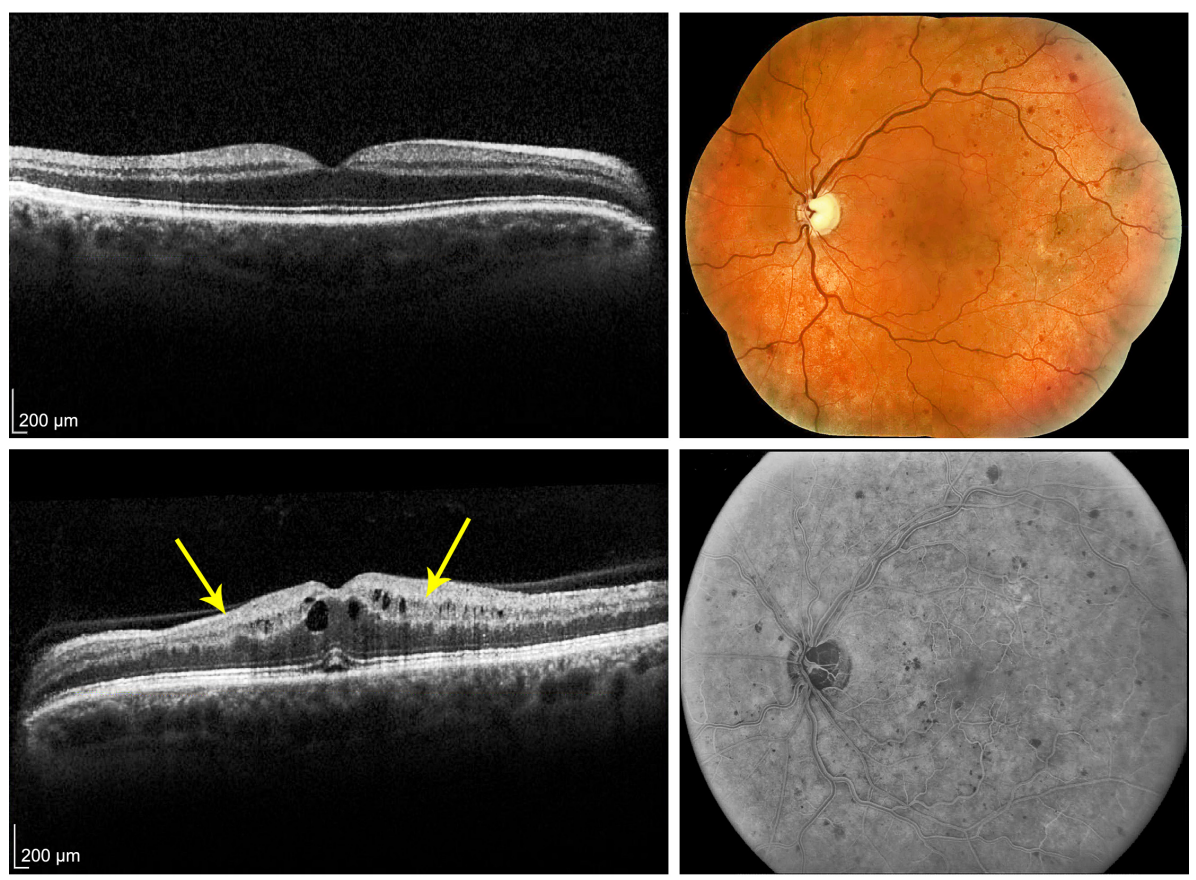

Figure I Grading standard used for a CRVO with none to mild ischemia (grade I).

Notes: Top left panel: the SD-OCT B-scan for the normal right eye is shown for comparison. Top right panel: color fundus photograph of the left eye with CRVO. The veins are dilated, and a few scattered dot hemorrhages are present in all four quadrants of the fundus with some in the macula. Best-corrected visual acuity was 20/40. There was no relative afferent pupillary defect. Bottom left panel: the SD-OCT B-scan of the left eye showing excellent delineation of the inner retinal layers (yellow arrows; each layer seen in the normal scan of the top left panel can be identified here). Mild cystoid macular edema is present. Bottom right panel: a mid-phase frame from the fluorescein angiogram of the left eye shows the absence of ischemic zones in the retina and the presence of mild fluorescein leakage.

Abbreviations: CRVO, central retinal vein occlusion; SD-OCT, spectral domain optical coherence tomography.
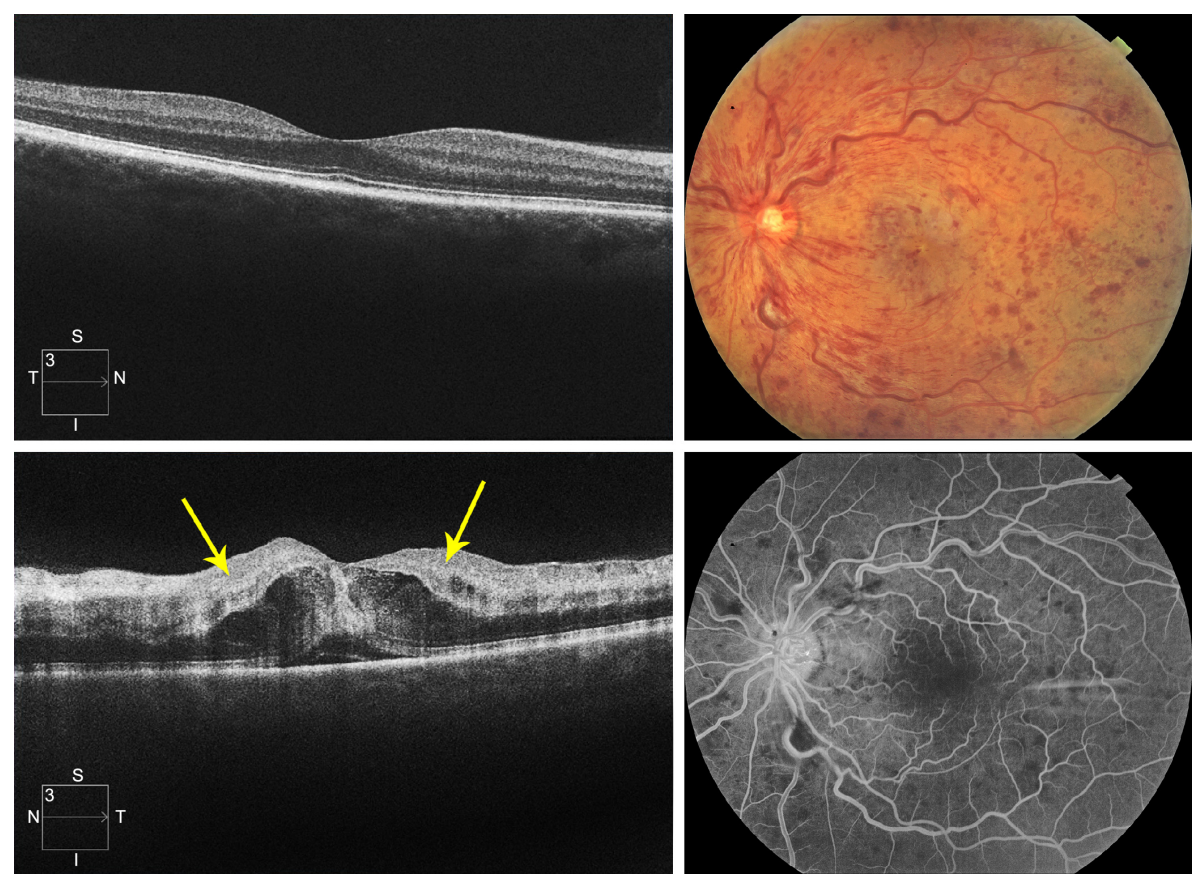

Figure 2 Grading standard used for a CRVO with moderate ischemia (grade 2).

Notes: Top left panel: the SD-OCT B-scan for the normal right eye is shown for comparison. Top right panel: color fundus photograph of the left eye with CRVO. The veins are more dilated than in the case with mild ischemia (Figure I), and more dot hemorrhages are present in all four quadrants of the fundus. The best-corrected visual acuity was 20/300. A moderate relative afferent pupillary defect was present. Bottom left panel: the SD-OCT B-scan of the left eye showing partial loss of delineation of the inner retinal layers and increased reflectivity (yellow arrows; compare to top left panel). Worse cystoid macular edema is present than in the case with mild ischemia and subretinal fluid is present, but neither factor was included in grading for ischemia. Bottom right panel: a mid-phase frame from the fluorescein angiogram of the left eye shows the absence of ischemic zones in the retina and the absence of fluorescein leakage.

Abbreviations: CRVO, central retinal vein occlusion; SD-OCT, spectral domain optical coherence tomography; N, nasal; S, superior; T, temporal; I, inferior. 

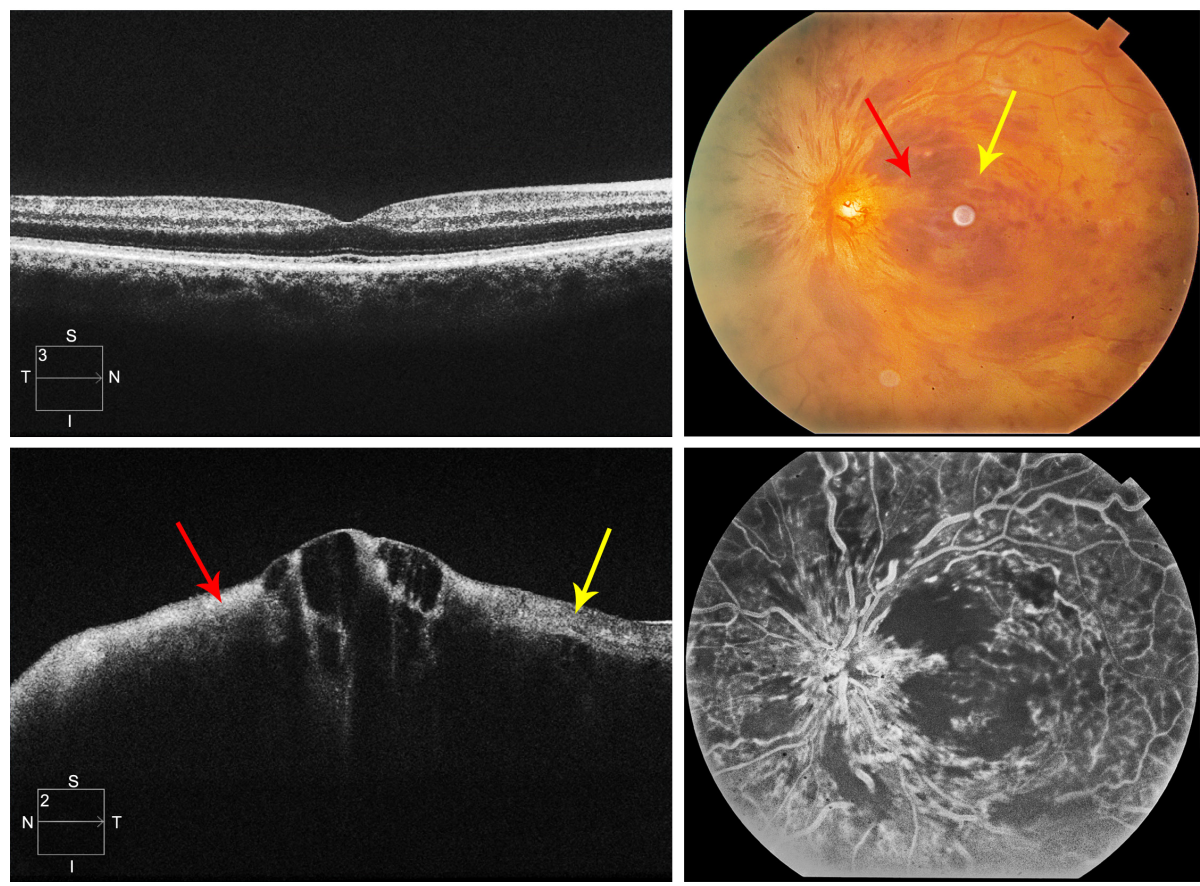

Figure 3 Grading standard used for a CRVO with severe ischemia (grade 3).

Notes: Top left panel: the SD-OCT B-scan for the normal right eye is shown for comparison. Top right panel: color fundus photograph of the left eye with CRVO. The veins are dilated and severe intraretinal hemorrhage is present in all four quadrants of the fundus. The red arrow indicates an area with nearly confluent intraretinal hemorrhage. The yellow arrow indicates an area relatively spared from intraretinal hemorrhage, which is the area for which the SD-OCT can be assessed without confounding from blood. The visual acuity was counting fingers at 2 feet. A marked relative afferent pupillary defect of the left eye was present. Bottom left panel: the SD-OCT B-scan of the left eye showing severe loss of delineation of the inner retinal layers and marked increase in reflectivity (yellow arrow; compares to top right panel). There is also increased reflectivity and loss of delineation of inner retinal layers at the red arrow, but in this case, the changes arise from a combination of the intraretinal blood and the ischemia. The region indicated by the yellow arrow is used for grading as it lacks the confounding inner retinal hemorrhage. Marked cystoid macular edema is present but this factor was not included in grading. Bottom right panel: a mid-phase frame from the fluorescein angiogram of the left eye shows zones of capillary nonperfusion and zones in which perfusion cannot be graded due to the presence of intraretinal hemorrhage.

Abbreviations: CRVO, central retinal vein occlusion; SD-OCT, spectral domain optical coherence tomography; N, nasal; S, superior; T, temporal; I, inferior.

Table 2 shows the distribution of baseline VAs, 12-month VAs, and change in VAs as a function of baseline SD-OCT ischemia grades. The median logarithm of the minimum angle of resolution (logMAR) VA at baseline was progressively worse as the baseline SD-OCT ischemia grade increased, but the trend was not statistically significant $(P=0.1246$, Kruskal-Wallis test). The median logMAR VA at 12-month follow-up progressively worsened as the baseline SD-OCT ischemia grade increased, but the trend was not statistically significant ( $P=0.1266$, Kruskal-Wallis test). There was no trend in distribution of 12-month change in logMAR visual acuities as a function of baseline SD-OCT ischemia grades $(P=0.5780$, Kruskal-Wallis test $)$.

The time course of logMAR VA as a function of baseline SD-OCT ischemia grades is shown in Figure 5.

Table 2 also shows the baseline CSMTs, 12-month CSMTs, and change in CSMTs as a function of baseline $\mathrm{SD}-\mathrm{OCT}$ ischemia grades. There was no trend in baseline CSMT as a function of ischemia grade $(P=0.1901$, Kruskal-Wallis test). For the 12-month CSMT, a trend was suggested with a more severe ischemia grade at baseline associated with a thicker 12-month CSMT, but the result did not reach statistical significance $(P=0.0931$, KruskalWallis test). For the 12-month change in CSMT, no trend relative to baseline ischemia was present $(P=0.4822$, Kruskal-Wallis test).

Although the prevalence of an RAPD at baseline increased with increasing baseline ischemia grade, the association did not reach statistical significance $(P=0.4096$, likelihood ratio test). There was no trend in the 12-month rate of development of ASNV with baseline ischemia grade ( $P=0.9040$, likelihood ratio test).

The presence or absence of a p-MLM sign was determined from the baseline SD-OCTs, and the correlation of the baseline ischemia grade was determined (Figure 6A). The proportion of eyes with the p-MLM sign did not vary significantly with respect to the baseline ischemia grade ( $P=0.8738$, likelihood ratio test). Similarly, the presence or absence of a PAMM sign was determined from the baseline SD-OCTs and the correlation of the baseline ischemia grade was determined (Figure 6B). The proportion of eyes with a PAMM sign was significantly greater in the eyes with severe 

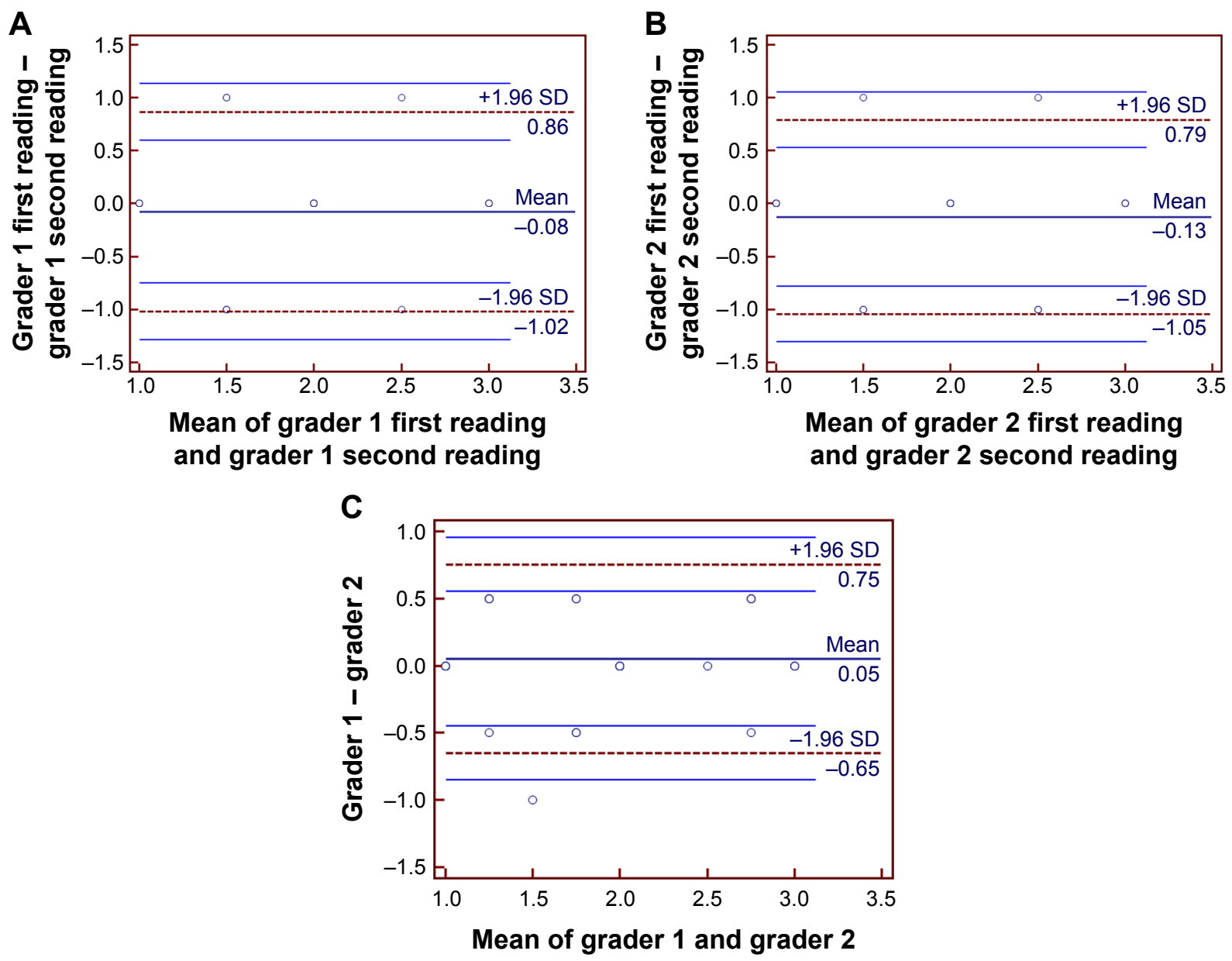

Figure 4 Bland-Altman plots of intragrader repeatability and intergrader reproducibility in the assessment of SD-OCT images in acute central retinal vein occlusion. Notes: (A) Bland-Altman plot for 2 gradings of the same set of 39 SD-OCT images performed by grader I. (B) Bland-Altman plot for 2 gradings of the same set of 39 SD-OCT images performed by grader 2. (C) Bland-Altman plot for gradings of graders I and 2 of the same set of 39 SD-OCT images. The dotted lines represent the limits of agreement. Abbreviations: SD, standard deviation; SD-OCT, spectral domain optical coherence tomography.

ischemia ( $P=0.0493$, likelihood ratio test). There was no significant association between having a p-MLM sign and a PAMM sign (Figure 6C; $P=0.2051$, Fisher's exact test). In some eyes, the SD-OCT signs of ischemia evolved. The PAMM sign occurred earlier and the p-MLM sign occurred later (Figure 7).

The numbers of interventions used in treating the eyes for macular edema and ASNV during the 12 months of follow-up are shown in Table 3. There was no difference in the number of interventions of any type across the grades of baseline ischemia.

\section{Discussion}

CRVO causes a higher resistance to blood flow through the retina. As the grade of the occlusion increases, flow begins to diminish and oxygenation of the inner retina is impaired,

Table I Characteristics of patients and eyes

\begin{tabular}{|c|c|c|c|c|c|}
\hline \multirow[t]{2}{*}{ Characteristic } & \multirow[t]{2}{*}{ Overall sample } & \multicolumn{3}{|l|}{ Grade of ischemia } & \multirow[t]{2}{*}{$P$-value } \\
\hline & & Mild (N=13) & Moderate $(\mathbf{N}=17)$ & Severe $(\mathbf{N}=9)$ & \\
\hline Age (mean, SD) & 70,13 & 68,11 & 69,15 & 76,11 & 0.2163 \\
\hline Gender (F:M) & $|8: 2|$ & $6: 7$ & $6: 11$ & $6: 3$ & 0.3076 \\
\hline Hypertension, N (\%) & $24(62)$ & $7(54)$ & II (65) & $6(67)$ & 0.7815 \\
\hline Diabetes, N (\%) & $8(2 I)$ & $5(38)$ & I (6) & $2(22)$ & 0.775 \\
\hline Primary open angle glaucoma, N (\%) & $5(13)$ & I (8) & $3(18)$ & $I(I I)$ & 0.7057 \\
\hline $\begin{array}{l}\text { Other macular conditions (number } \\
\text { without: list of other conditions, } \mathrm{N} \text { ) }\end{array}$ & 32: DME (3), ERM (2), AMD (2) & 10: DME (2), ERM (I) & I4: ERM (I), AMD (2) & 8: DME (I) & 0.3378 \\
\hline
\end{tabular}

Abbreviations: AMD, age-related macular degeneration; DME, diabetic macular edema; ERM, epiretinal membrane; F, female; M, male; N, number of patients; $\mathrm{SD}$, standard deviation. 
Table 2 Distribution of baseline characteristics and outcomes by baseline grade of ischemia

\begin{tabular}{|c|c|c|c|c|}
\hline \multirow[t]{2}{*}{ Characteristic } & \multicolumn{3}{|c|}{ Baseline grade of ischemia } & \multirow[t]{2}{*}{$P$-value } \\
\hline & Mild & Moderate & Severe & \\
\hline $\mathrm{N}$ & 13 & 17 & 9 & \\
\hline Baseline VA & $0.480(0.300,0.65 \mathrm{I})$ & $0.602(0.300,1.088)$ & $0.903(0.521,1.626)$ & $0.1246 *$ \\
\hline I2-month VA & $0.200(0.100,0.449)$ & $0.200(0.100,1.000)$ & $0.480(0.300,1.577)$ & $0.1266 *$ \\
\hline I2-month change in VA & $-0.300(-0.439,0.049)$ & $-0.100(-0.410,0.300)$ & $0.000(-0.400,0.300)$ & $0.5780 *$ \\
\hline Baseline CSMT & $55 I(407,593)$ & $659(421,708)$ & $596(4 \mid 4,736)$ & $0.1901 *$ \\
\hline I2-month CSMT & $332(231,429)$ & $314(25 I, 446)$ & $495(330,694)$ & $0.0931 *$ \\
\hline I2-month change in CSMT & $-179(-95,-272)$ & $-280(-6 \mid,-345)$ & $-124(194,-32 \mid)$ & $0.4822^{*}$ \\
\hline Prevalence of baseline RAPD (\%) & 15.4 & 23.5 & 44.4 & $0.4096 * *$ \\
\hline Incidence of ASNV over 12 months (\%) & 15.4 & 17.6 & 8.9 & $0.9040 * *$ \\
\hline
\end{tabular}

Notes: Entries are medians (interquartile range). Grades of ischemia are I (mild), 2 (moderate), and 3 (severe). $* P$-values reflect Kruskal-Wallis testing. $* * P$-values reflect likelihood ratio testing.

Abbreviations: ASNV, anterior segment neovascularization; CSMT, central subfield mean thickness; N, number of patients; RAPD, relative afferent pupillary defect; VA, visual acuity.

a situation termed ischemia. Whereas, earlier work placed this pathophysiologic phenomenon into two categories nonischemic and ischemic, a more recent refinement of the concept emphasizes continuous gradation as reflected in the concentration of VEGF in the extracellular fluid of the retina and indirectly in the vitreous.

Clinically, assessing ischemia is important to determine the risk of subsequently developing ASNV and NVG. The clinical assessment is the basis for advising the patient on a follow-up schedule: when the risk is high, follow-up intervals are shorter. All previous methods for assessing ischemia, including VA, visual field, FA, RAPD, and global ERG, have been found to have too many false negatives to excuse the clinician from frequent follow-up. Compounding the problem is the tendency of CRVOs with lesser ischemia to evolve into CRVOs of greater ischemia. In the era of

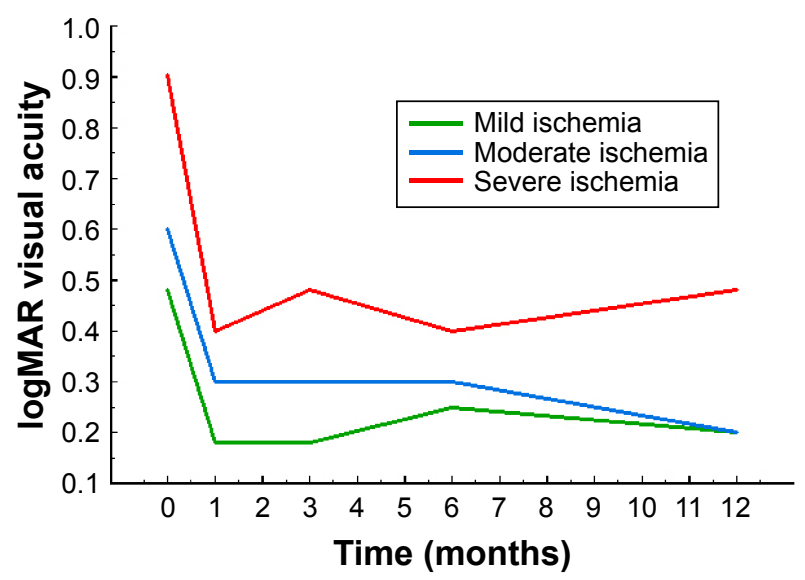

Figure $\mathbf{5}$ The time course of logMAR visual acuity graphed as a function of time in months and stratified by baseline ischemia grade as graded from SD-OCT images.

Note: The lines represent grade I ischemia subgroup (green line), the grade 2 ischemia subgroup (blue line), and grade 3 ischemia subgroup (red line).

Abbreviations: logMAR, logarithm of the minimum angle of resolution; SD-OCT, spectral domain optical coherence tomography. classifying CRVOs as nonischemic and ischemic, the conversion rate was estimated to be $16 \%$ in 4 months and $34 \%$ in 3 years. ${ }^{8}$

With the advent of SD-OCT, 3 groups have described SD-OCT signs of ischemia in acute CRVO, but data on prognostic accuracy of these signs for later ASNV have been published for only one (the p-MLM sign), and the interrelationships of the signs have not been explored.

We found that none of the signs was reliably predictive of later ASNV, and that the signs did not reliably correlate with each other or with other signs such as baseline VA or presence of RAPD. We did not measure global ERG or FA routinely, and have no data to report on correlations with those testing modalities. Fluorescein angiographic indices of capillary nonperfusion are beset with problems of intergrader variability and subjectivity. ${ }^{9,10}$ In one study, combined intragrader and intergrader replicate coefficients of variation were $21.2 \%$ for CRVO. ${ }^{9}$ The prognostic accuracy of global ERG has likewise been poor, and it has not been widely adopted in the workup of patients with CRVO.

Our data showed no correlation of any intervention for macular edema with baseline ischemia grade. Treatment of macular edema with anti-VEGF injections was as common in eyes with mild ischemia as it was in eyes with severe ischemia. The frequent injection of anti-VEGF drugs not only treated the macular edema but also probably masked any underlying associations of ASNV and NVG with baseline ischemia. It is known that anti-VEGF drugs reduce the levels of intravitreal VEGF and reduce rates of enlargement of areas of capillary nonperfusion. ${ }^{11}$ The area of retinal nonperfusion can increase with reduction in frequency of anti-VEGF drug injection; the possibility should stimulate continued vigilance and slow tapering in frequency of follow-up. 

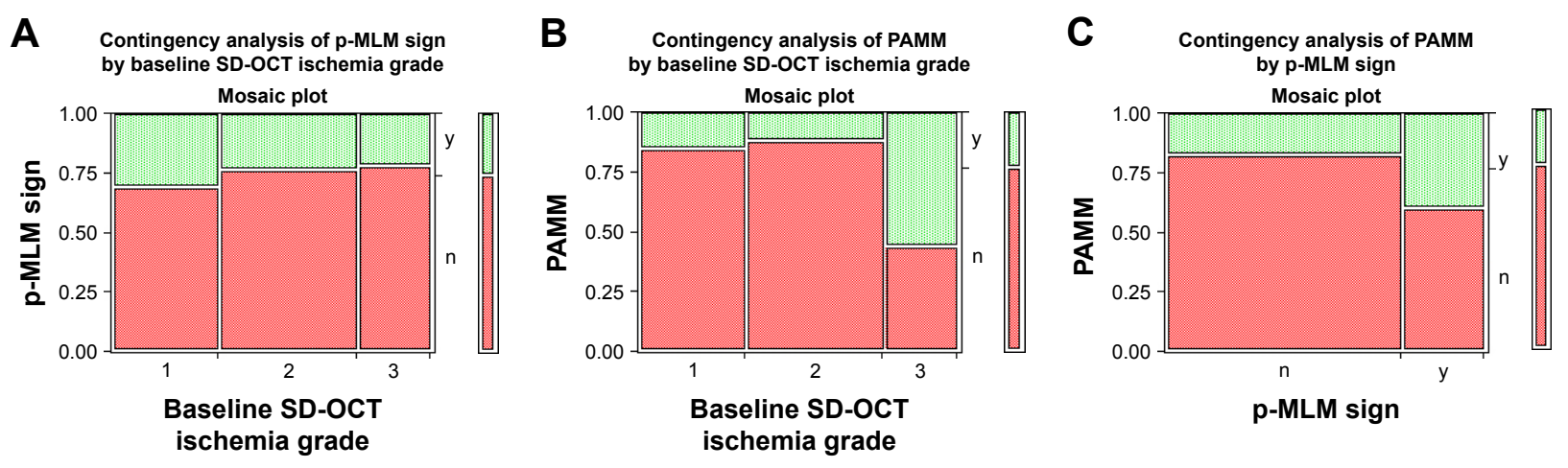

Figure 6 Graphs of the relationships of three SD-OCT signs to each other.

Notes: In each panel, for each grade of ischemia, the green bar (y) indicates the proportion of eye that had the sign, and the red bar ( $\mathrm{n}$ ) indicates the proportion of eyes without the sign. (A) Graph of the proportion of eyes with the P-MLM sign as a function of the baseline grade of ischemia by SD-OCT grade. (B) Graph of the proportion of eyes with the PAMM sign as a function of the baseline grade of ischemia by SD-OCT grade. (C) Graph of the proportion of eyes with the PAMM sign as a function of the presence or absence of a p-MLM sign.

Abbreviations: PAMM, paracentral acute middle maculopathy; P-MLM, prominent middle-limiting membrane; SD-OCT, spectral domain optical coherence tomography.

Others have also described inner nuclear layer and superficial outer plexiform layer hyperreflectivity along the paramacular retinal venules in ostensibly nonischemic CRVO, terming the phenomenon patchy ischemic retinal whitening, ${ }^{12}$ perivenular retinal whitening, ${ }^{13}$ and paramacular acute middle maculopathy. ${ }^{5}$ Schröder et al described inner retinal atrophy in eyes with $\mathrm{CRVO}$ and poor VA following the resolution of baseline macular edema. ${ }^{14}$ Ebneter et al have developed a mouse model that simulates the clinical correlations that we have described. In acute CRVO in the mouse, the OCT changes are described as follows: "Hyperreflectivity with washed-out retinal structures and thickening of the retina are observed within the first few days". Subsequently, the inner retina thins and becomes hypocellular. ${ }^{15}$ We witnessed cases in which PAMM, p-MLM, and inner retinal layer hyperreflectivity were seen in isolation, together in pairs, or all present simultaneously in different regions. In addition, PAMM can evolve over time into p-MLM. Further study will be needed to understand how these manifestations of ischemia in CRVO are related spatially within the macula and longitudinally over time. OCT angiography has the potential to provide further insight into retinal ischemia in CRVO,
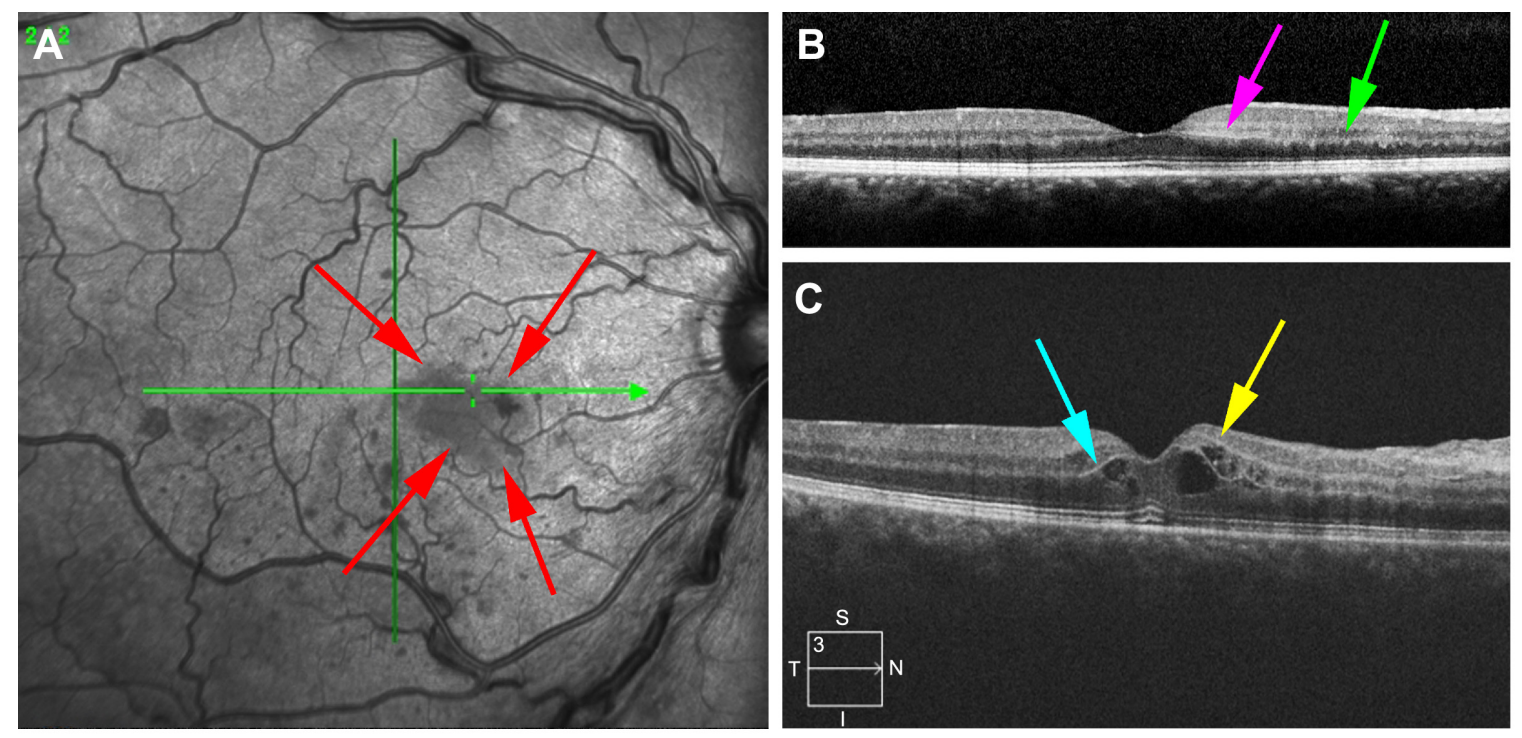

Figure 7 Evolution of SD-OCT signs of ischemia over time.

Notes: (A) Near infrared reflectance imaging photograph of the right fundus of a 60-year-old woman with an acute central retinal vein occlusion of the right eye. (A) Red arrows indicate the hyporeflective lesion indicative of PAMM. Green arrow indicates the location of the scan in panel B. (B) Radial line scan from the SD-OCT showing the location of PAMM (purple arrow). The INL is hyperreflective compared to the adjacent normal region (green arrow). (C) Radial line scan from the SD-OCT I7 days after the image of $B$ showing resolution of PAMM, development of cystoid macular edema, and new development of the $\mathrm{p}$-MLM sign (turquoise arrow) at the outer border of the INL. In addition, a new sign, a hyperreflective line at the inner border of the INL similar to the p-MLM sign, is seen (yellow arrow).

Abbreviations: INL, inner nuclear layer; PAMM, paracentral acute middle maculopathy; P-MLM, prominent middle-limiting membrane; SD-OCT, spectral domain optical coherence tomography. 
Table 3 Number of interventions over 12 months in acute central retinal vein occlusion

\begin{tabular}{|c|c|c|c|c|}
\hline \multirow[t]{2}{*}{ Outcome } & \multicolumn{3}{|c|}{ Baseline ischemia status by SD-OCT grading } & \multirow[t]{2}{*}{$P$-value } \\
\hline & I (mild) & 2 (moderate) & 3 (severe) & \\
\hline Number of anti-VEGF injections & $6(5,9), 11$ & $6(4,9), 12$ & $6(3,7), 12$ & 0.8540 \\
\hline Number of PRPs & $0(0,0), 0$ & $0(0,0), \mathrm{I}$ & $0(0,0), 1$ & 0.5094 \\
\hline Number of grid laser treatments & $0(0,0), 1$ & $0(0,0), 0$ & $0(0,0), 0$ & 0.3679 \\
\hline Number of intravitreal steroid injections & $0(0,0), 1$ & $0(0,0), I$ & $0(0,0), 2$ & 0.9238 \\
\hline $\begin{array}{l}\text { Incisional glaucoma procedures (total cases: } \\
\text { number of cases of specified ischemia grade) }\end{array}$ & $1: 13$ & $\mathrm{I}: 17$ & $0: 9$ & $0.57 / 2$ \\
\hline $\begin{array}{l}\text { Laser cyclophotocoagulation (total cases: } \\
\text { number of cases of specified ischemia grade) }\end{array}$ & $1: 13$ & $0: 17$ & $1: 9$ & 0.2941 \\
\hline
\end{tabular}

Notes: The number of anti-VEGF injections applied for 12 months following the diagnosis of central retinal vein occlusion. Entries are medians followed by interquartile ranges in parentheses, in turn, followed by the maximum number for the subgroup. The number of anti-VEGF injections is the sum of bevacizumab, ranibizumab, and aflibercept injections. The number of intravitreal steroid injections is the sum of intravitreal triamcinolone and dexamethasone sustained release device injections.

Abbreviations: PRPs, panretinal photocoagulations; SD-OCT, spectral domain optical coherence tomography; VEGF, vascular endothelial growth factor.

but is also limited by the blocking properties of intraretinal hemorrhage and the technical limitations of scanning the retinal periphery. ${ }^{16}$

\section{Conclusion}

In summary, no single clinical or ancillary test sign has proven to be reliable for assessing ischemia in acute CRVO. This may in part reflect the subjectivity in grading, and may reflect the fact that ischemia in CRVO is dynamic. We suggest that increased reflectivity of the inner retina in acute CRVO provides evidence that inner retinal oxygenation is inadequate to maintain metabolic homeostasis and normal retinal transparency. It does not imply that retinal atrophy is inevitable as witnessed by cases having increased inner retinal reflectivity that subsequently go on to recover definition of the retinal layers later. The SD-OCT signs of inner retinal ischemia in acute CRVO help the clinician understand the pathophysiology, but cannot substitute for frequent follow-up in the first year until the risk of ASNV falls enough that intervals can be lengthened. Even though SD-OCT is an excellent imaging technique in patients with acute CRVO, our results indicate that there is still no reliable imaging characteristic on baseline SD-OCT that prognosticates subsequent neovascular outcomes.

\section{Disclosure}

The authors report no conflicts of interest in this work.

\section{References}

1. Hayreh SS, Podhajsky PA, Zimmerman MB. Natural history of visual outcome in central retinal vein occlusion. Ophthalmology. 2011;118(1): 119-133.

2. Servais GE, Thompson HS, Hayreh SS. Relative afferent pupillary defect in central retinal vein occlusion. Ophthalmology. 1986;93(3): 301-303.
3. Sabates R, Hirose T, McMeel JW. Electroretinography in the prognosis and classification of central retinal vein occlusion. Arch Ophthalmol. 1983;101(2):232-235.

4. Ko J, Kwon OW, Byeon SH. Optical coherence tomography predicts visual outcome in acute central retinal vein occlusion. Retina. 2014;34(6): $1132-1141$.

5. Rahimy E, Sarraf D, Dollin M, Pitcher JD, Ho AC. Paracentral acute middle maculopathy in nonischemic central retinal vein occlusion. Am J Ophthalmol. 2014;158(2):372-380.

6. Browning DJ. Retinal Vein Occlusions. Evidence Based Management. New York, NY: Springer; 2012:366.

7. Bland JM, Altman DG. Statistical methods for assessing agreement between two methods of clinical measurement. Lancet. 1986;1(8476): 307-310.

8. The Central Vein Occlusion Study Group. Baseline and early natural history report. Arch Ophthalmol. 1993;111(8):1087-1095.

9. Tsui I, Williams BK Jr, Kok YO, Heilweil G, Schwartz SD. Reliability of ischemic index grading in common retinal vascular diseases. Ophthalmic Surg Lasers Imaging Retina. 2015;46(6):618-625.

10. Welch JC, Augsburger JJ. Assessment of angiographic retinal capillary nonperfusion in central retinal vein occlusion. Am J Ophthalmol. 1987;103(6):761-766.

11. Mir TA, Kerani S, Hafiz G, et al. Changes in retinal nonperfusion associated with suppression of vascular endothelial growth factor in retinal vein occlusion. Ophthalmology. 2016;123(3):625-634.

12. Browning DJ. Patchy ischemic retinal whitening in acute central retinal vein occlusion. Ophthalmology. 2002;109(11):2154-2159.

13. Paques M, Gaudric A. Perivenular macular whitening during acute central retinal vein occlusion. Arch Ophthalmol. 2003;121(10): 1488-1491.

14. Schröder K, Ackermann P, Brachert M, Bairov S, Geerling G, Guthoff R. Does OCT morphology provide indications for prognosis of $\mathrm{VA}$ after venous occlusion?: SD-OCT analysis in retinal vein occlusion before and after resolution of initial macular edema. Ophthalmologe. 2016;113(6):510.

15. Ebneter A, Agca C, Dysli C, Zinkernagel MS. Investigation of retinal morphology alterations using spectral domain optical coherence tomography in a mouse model of retinal branch and central retinal vein occlusion. PLoS One. 2015;10:e119046.

16. Kimura M, Nozaki M, Yoshida M, Ogura Y. Wide-field optical coherence tomography angiography using extended field imaging technique to evaluate nonperfusion area in retinal vein occlusion. Clin Ophthalmol. 2016;10:1291-1295. 


\section{Publish your work in this journal}

Clinical Ophthalmology is an international, peer-reviewed journal covering all subspecialties within ophthalmology. Key topics include: Optometry; Visual science; Pharmacology and drug therapy in eye diseases; Basic Sciences; Primary and Secondary eye care; Patien Safety and Quality of Care Improvements. This journal is indexed on

Submit your manuscript here: http://www.dovepress.com/clinical-ophthalmology-journal
Dovepress

PubMed Central and CAS, and is the official journal of The Society of Clinical Ophthalmology (SCO). The manuscript management system is completely online and includes a very quick and fair peer-review system, which is all easy to use. Visit http://www.dovepress.com/ testimonials.php to read real quotes from published authors. 